\title{
New pharmaceuticals in inflammatory bowel disease
}

\author{
Michał Łodyga ${ }^{1}$, Piotr Eder², Witold Bartnik², Maciej Gonciarz', Maria Kłopocka5 ${ }^{5}$ Krzysztof Linke², \\ Ewa Małecka-Panas ${ }^{6}$, Piotr Radwan, Grażyna Rydzewska ${ }^{1,8}$ \\ ${ }^{1}$ Department of Internal Medicine and Gastroenterology with IBD Subdivision, Central Clinical Hospital of the Ministry \\ of the Interior, Warsaw, Poland \\ ${ }^{2}$ Department of Gastroenterology, Human Nutrition and Internal Diseases, Poznan University of Medical Sciences, \\ Poznan, Poland \\ ${ }^{3}$ Department of Gastroenterology and Hepatology, Medical Centre for Postgraduate Education, Warsaw, Poland \\ ${ }^{4}$ Department of Gastroenterology, St. Barbara Provincial Specialist Hospital, Sosnowiec, Poland \\ ${ }^{5}$ Gastroenterology Nursing Unit, Centre for Therapeutic Endoscopy, University Hospital No 2, Collegium Medicum in Bydgoszcz, \\ Nicolaus Copernicus University in Torun, Poland \\ ${ }^{6}$ Department of Gastrointestinal Tract Diseases, Medical University of Lodz, Lodz, Poland \\ 7Department Gastroenterology and Endoscopy, Medical University of Lublin, Lublin, Poland \\ ${ }^{8}$ Faculty of Health Sciences, Jan Kochanowski University, Kielce, Poland
}

Prz Gastroenterol 2015; 10 (2): 57-60

DOI: $10.5114 / p g .2015 .52702$

Key words: Crohn's disease, ulcerative colitis, budesonide MMX, golimumab, vedolizumab.

Address for correspondence: Prof. Grażyna Rydzewska MD, PhD, Department of Internal Medicine and Gastroenterology with IBD Subdivision, Central Clinical Hospital of the Ministry of the Interior, 137 Woloska St, 02-507 Warsaw, Poland, e-mail: grazyna.rydzewska@cskmswia.pl

\begin{abstract}
This paper complements the previously published Guidelines of the Working Group of the Polish Society of Gastroenterology and former National Consultant in Gastroenterology regarding the management of patients with Crohn's disease and ulcerative colitis. Attention was focused on the new pharmaceutical recently registered for inflammatory bowel disease treatment.
\end{abstract}

\section{Introduction}

The treatment of patients with inflammatory bowel disease (IBD), namely Crohn's disease (CD) and ulcerative colitis (UC), may be associated with significant problems due to the common insufficient response to therapeutic regimens or to the loss of response in the course of long-term treatment. Despite extensive studies carried out at many centres worldwide, causal treatment of this group of diseases is still unavailable. However, advances made in recent years have allowed the introduction into clinical practice of some novel products characterised by better efficacy and more favourable safety profiles. This study complements the previously published Guidelines of the Working Group of the Polish Society of Gastroenterology and former National Consultant in Gastroenterology regarding the management of patients with Crohn's disease and ulcerative colitis $[1,2]$.

\section{Budesonide}

Budesonide $M M X$ at a single daily dose of $9 \mathrm{mg}$ is efficient in inducing remission in patients with mild to moderate active ulcerative colitis where remission was not achieved after administration of mesalazine or sulfasalazine, or who are intolerant to 5-ASA drugs. Due to its favourable safety profile, budesonide MMX should be considered as the firstchoice steroid treatment.

Budesonide MMX (Multi Matrix System) is an oral glucocorticosteroid that, due to its unique formulation, is uniformly released along the entire large intestine. This makes it appropriate to use in both left-sided colitis and pancolitis. In addition, low bioavailability of budesonide of nearly $90 \%$, due to its first pass ef- 
fect in the liver leading to the formation of inactive or nearly inactive metabolites, minimises the adverse systemic effects typical for this group of drugs [3]. Clinical studies conducted to date have demonstrated the efficacy of this budesonide formulation in inducing remission in patients with mild to moderate active ulcerative colitis, previously treated or untreated with mesalazine formulations. In the CORE I and CORE II studies $[4,5]$ the drug was used for a period of 8 weeks, and in the case of poor effects the treatment period was extended to 16 weeks. The endpoints of these studies consisted of clinical, colonoscopic, and histological remission that offered better chances of the maintenance of longer symptom-free periods [6]. The efficacy of two doses of budesonide MMX (9 and $6 \mathrm{mg} /$ day) was compared with mesalazine (2.4 g/day), Entocort EC (9 mg/ day), and placebo. A statistically significant increase in the rate of full clinical, colonoscopic, and histological remission was observed in the $9 \mathrm{mg}$ /day budesonide MMX group as compared to placebo. A beneficial therapeutic effect was observed in both left-sided colitis and pancolitis. According to clinical study-confirmed indications, budesonide MMX should be used in case of failure of previous mesalazine treatment; it is also acceptable as the treatment of first choice [7]. The introduction of budesonide MMX led to a change in the treatment algorithm for mild to moderate active UC. In the case of indications for steroid therapy, it is recommended that treatment with budesonide $M M X$ be initiated before other systemic drugs of this group [7]. Budesonide MMX is a safe drug that is free of systemic side effects typical for other glucocorticoids. Although reduction in the levels of endogenous cortisol was observed during the treatment in clinical studies, the values remained within normal limits (also in the case of treatment duration being extended to 16 weeks) $[4,5,7]$. Therefore, tapering of the drug is not required. Currently, clinical studies are ongoing regarding the use of budesonide $M M X$ in maintenance remission in UC (treatment extended to 12 months). As demonstrated by an interim safety data analysis, long-term use of the drug does not lead to further reduction in cortisol levels $[8,9]$. However, the place of budesonide MMX in therapeutic algorithms as compared to other drugs used in the treatment of UC, particularly immunosuppressive and biological agents, remains undetermined.

Previous experience gained with use of budesonide (Entocort EC) in Crohn's disease, with comparable safety profiles of both forms of budesonide (head-to-head registration trials) $[3,4]$, justifies the use of both drugs as combination treatment in selected cases; however, further clinical studies are required so that more explicit recommendations may be formulated.

\section{Vedolizumab}

\section{Vedolizumab is indicated in the case of the lack of efficacy or intolerance to conventional treat- ment and/or treatment with anti-TNF- $\alpha$ agents, in patients with moderate to severe UC or CD.}

Besides anti-TNF- $\alpha$ biological agents, another novel and promising group of medications consists of anti-adhesive compounds that inhibit the migration of leukocytes by blocking the adhesive molecules in vascular epithelium or integrin molecules at the surface of leukocytes. To date, the use of these substances was restricted due to the risk of progressive multifocal leukoencephalopathy (PML) in patients treated with a non-selective anti-integrin $\alpha 4$ antibody, natalizumab [10]. Blocking the $\alpha 4$ subunit inhibits the inflow of leukocytes into the gastrointestinal tract and, by means of inhibition of binding to the vascular cell adhesion molecule 1 (VCAM-1), into all other systems, including the central nervous system.

Vedolizumab is a humanised monoclonal antibody that binds integrin $\alpha 4 \beta 7$ molecules present on the membranes of leukocytes (particularly T-cells), thus blocking interactions of these molecules with mucosal addressin cell adhesion molecules-1 (MadCAM-1) on epithelial cells. Integrin $\alpha 4 \beta 7$ is expressed on the surface of cells that migrate into blood and lymphatic vessels of the gastrointestinal tract, while MAdCAM-1 is expressed mainly in intestinal epithelial cells; therefore, inhibition of leukocyte migration following administration of vedolizumab occurs in a selective manner and plays a key role in selective inhibition of inflammation within the intestine.

The drug does not bind and does not inhibit the function of integrin $\alpha 4 \beta 1$; it also does not inhibit interactions of integrin $\alpha 4$ with VCAM-1, thus eliminating the risk of adverse systemic effects, particularly PML [10]. The drug received a positive opinion of the Committee for Medicinal Products for Human Use (CHMP) of the European Medicines Agency (EMA) and was registered in May 2014 for the induction and maintenance treatment of adult patients with moderately to severely active UC or CD.

These decisions were underpinned by the results of the GEMINI I (UC) as well as GEMINI II and GEMINI III (CD) clinical studies [11-13]. The clinical program for vedolizumab was the largest clinical program conducted to date in IBD patients, with a total population of 2700 subjects. Currently, an open-label GEMINI LTS (long-term safety) trial is underway. Included in the studies are patients with moderately to severely 
active UC or CD in whom the standard treatment and/or anti-TNF- $\alpha$ treatment has proven inefficient or poorly tolerated. The efficacy endpoints in the GEMINI I study were the percentage of patients with clinical improvement at treatment week 6 and the percentage of patients with clinical remission being maintained after 52 weeks of maintenance therapy. Vedolizumab was shown to be statistically superior to placebo in terms of inducing clinical response ( $47 \%$ vs. $26 \%$ ) as well as maintaining remission. A statistically significant difference was also observed in the percentage of patients with clinical remission and mucosal healing at treatment week 6 [11].

The endpoints of the GEMINI II study included: the percentage of patients with clinical improvement at treatment week 6 and the percentage of patients with clinical remission being maintained after 52 weeks of maintenance therapy. Induction treatment with vedolizumab was shown to be statistically superior to placebo in terms of clinical remission (14.5\% vs. $6.8 \%$ ) while the benefit in terms of clinical response was not statistically significant [12].

The GEMINI II study was supplemented by the GEMINI III study, which assessed the efficacy of vedolizumab in the induction of remission as measured at treatment weeks 6 and 10 in patients after previous failure of anti-TNF- $\alpha$ therapy [13]. At week 6 , vedolizumab was more effective than placebo in terms of inducing clinical response (39\% vs. $22 \%$ ) while a clear, statistically significant superiority of the drug in terms of the achievement of clinical remission was observed as late as at treatment week 10 (27\% vs. 12\%) [13].

Vedolizumab is well tolerated and the rate of adverse effects observed in the studies conducted to date was similar to that in the placebo groups. Particularly important from the standpoint of treatment safety is the minimised systemic immunosuppression. No cases of PML or increased incidence of malignancies have been observed to date $[14,15]$.

Registration indications of vedolizumab include moderate or severe exacerbations of $U C$ and $C D$ in adults in the case of failure or loss of efficacy of conventional treatment and /or anti-TNF- $\alpha$ treatment.

Considering the availability and costs of the treatment, a panel of experts is of the opinion that vedolizumab should be currently used in Poland in patients with documented lack of efficacy or intolerance to both conventional and anti-TNF- $\alpha$ treatment. Further clinical studies are required for vedolizumab to be recommended as an alternative to TNF- $\alpha$ antagonists.

The drug is administered in intravenous infusions at a dose of $300 \mathrm{mg}$ in weeks 0, 2, and 6 (induction of remission) and subsequently every 8 weeks (mainte- nance treatment). The efficacy of induction treatment is performed at treatment week 10; in the case of no effect, treatment discontinuation should be considered. If efficacy is lost during the maintenance treatment, one may consider shortening of the interval between successive doses to 4 weeks.

In CD patients with no distinct clinical improvement after three doses of induction treatment, another dose may be administered at week 10 and treatment efficacy may be assessed at week 14 .

\section{Golimumab}

\section{Golimumab is effective in the treatment of moder- ate to severe ulcerative colitis in patients resistant or intolerant to the standard treatment.}

Golimumab, a humanised monoclonal antibody, is another biological anti-TNF- $\alpha$ agent registered in the treatment of moderately to severely active UC (after infliximab and adalimumab).

A total of 1064 patients with failure of or intolerance to previous standard treatment were included in a clinical trial to assess the induction of remission using golimumab (PURSUIT-Induction) [16]. The first part of this trial consisted of a phase II study aimed at determination of the therapeutic dose of the drug. The second part was a phase III study to assess the efficacy of golimumab administered subcutaneously once every 2 weeks at doses of 200/100 mg or 400/200 mg, as compared to placebo. Clinical response after 6 weeks of treatment was achieved in $51-55 \%$ of patients receiving golimumab as compared to $30 \%$ of patients in the placebo group. Statistically significant differences in favour of both doses of golimumab as compared to placebo were also demonstrated when assessing clinical remission, mucosal healing, and quality-oflife improvement [16]. In the maintenance phase (PURSUIT-Maintenance), clinical response at week 54 was maintained in $47 \%$ of patients treated with the dose of $50 \mathrm{mg}$ and in $50 \%$ of patients treated with the dose of $100 \mathrm{mg}$ administered once every 4 weeks, as compared to $31 \%$ of patients in the placebo group [17].

In clinical practice, the recommended initial dose is $200 \mathrm{mg}$, followed by $100 \mathrm{mg}$ in the second week and then 50 or $100 \mathrm{mg}$ depending on body weight (100 mg for individuals weighing more than $80 \mathrm{~kg}$ ) administered subcutaneously every 4 weeks. Clinical assessment is conducted after the fourth dose of the drug to determine whether maintenance treatment should be continued in patients with good response to the induction treatment. 
Adverse effects observed in golimumab treatment groups are similar to those observed in the case of other TNF- $\alpha$ blockers, namely severe infections, including tuberculosis and opportunistic infections $[16,17]$. All recommendations valid for this group of drugs should be observed both when qualifying patients for the treatment and when continuing with the treatment.

One of the advantages of golimumab is its low immunogenicity, leading to a lower risk of systemic and local hypersensitivity reactions after administration as well as with lower anti-drug antibody production compared to other anti-TNF- $\alpha$ drugs. At the end of the 54week maintenance treatment, antibodies were detected in $3 \%$ of patients, including only $1 \%$ of patients concomitantly receiving immunosuppressive therapy [17]. Golimumab treatment may prove effective in cases of intolerance to other anti-TNF- $\alpha$ drugs or in cases of secondary intolerance to these drugs due to the formation of antibodies.

\section{References}

1. Łodyga M, Eder P, Bartnik W, et al. Guidelines for the management of Crohn's disease. Recommendations of the Working Group of the Polish National Consultant in Gastroenterology and the Polish Society of Gastroenterology. Prz Gastroenterol 2012; 7: 317-38.

2. Eder P, Łodyga M, Łykowska-Szuber L, et al. Guidelines for the management of ulcerative colitis. Recommendations of the Working Group of the Polish National Consultant in Gastroenterology and the Polish Society of Gastroenterology. Prz Gastroenterol 2013; 8: 1-20.

3. Edsbacker S, Bengtsson B, Larsson P, et al. A pharmacoscintigraphic evaluation of oral budesonide given as controlled-release (Entocort) capsules. Aliment Pharmacol Ther 2003; 17: 525-36.

4. Sandborn WJ, Travis S, Moro L, et al. Once-daily budesonide $M M X(R)$ extended-release tablets induce remission in patients with mild to moderate ulcerative colitis: results from the randomized CORE I study. Gastroenterology 2012; 143: 1218-26.

5. Travis SP, Danese S, Kupcinskas L, et al. Once-daily budesonide MMX in active, mild-to-moderate ulcerative colitis: results from the randomized CORE II study. Gut 2014; 63: 433-41.

6. Peyrin-Biroulet L, Ferrante M, Magro F, et al.; Scientific Committee of the European Crohn's and Colitis Organization. Results from the 2nd Scientific Workshop of the ECCO. I: impact of mucosal healing on the course of inflammatory bowel disease. J Crohns Colitis 2011; 5: 477-83.

7. Danese S, Siegel CA, Peyrin-Biroulet L. Inegrating budesonide$M M X$ into treatment algorithms for mild-to-moderate ulcerative colitis. Aliment Pharmacol Ther 2014; 39: 1095-103.

8. Lichtenstein GR, Danese S, Ballard ED, et al. Effect of budesonide MMX $6 \mathrm{mg}$ on the hypothalamic-pituitary-adrenal (HPA) axis in patients with ulcerative colitis: results from a phase III, 12 month safety and extended use study. Gastroenterology 2012; 142 (Suppl. 1): S785.
9. Travis S, Danese S, Ballard ED, et al. Safety analysis of budesonide MMX 6 mg used for the maintenance of remission in patients with ulcerative colitis: results from a phase III, 12 month safety and extended use study. Gastroenterology 2012; 142 (Suppl. 1): S566-7.

10. Chandar AK, Singh S, Murad MH, et al. Efficacy and safety of natalizumab and vedolizumab for the management of Crohn's disease: a systematic review and meta-analysis. Inflamm Bowel Dis 2015; 21: 1695-708.

11. Feagan B, Rutgeerts P, Sands B, et al. Vedolizumab as induction and maintenance therapy for ulcerative colitis. N Engl J Med 2013; 369: 699-710.

12. Sandborn W, Feagan B, Rutgeerts $P$, et al. Vedolizumab as induction and maintenance therapy for Crohn's disease. N Engl J Med 2013; 369: 711-21.

13. Sands B, Feagan B, Rutgeerts P, et al. Effects of vedolizumab induction therapy for patients with Crohn's disease in whom tumor necrosis factor antagonist treatment failed. Gastroenterology 2014; 147: 618-27.

14. Bryant RV, Sandborn WJ, Travis S. Introducing vedolizumab to clinical practice: who, when, and how? J Crohns Colitis 2015; 9: 356-66.

15. Gilroy L, Allen PB. Is there a role for vedolizumab in the treatment of ulcerative colitis and Crohn's disease? Clin Expert Gastroenterol 2014; 7: 163-72.

16. Sandborn WJ, Feagan BG, Marano C, et al. Subcutaneous golimumab induces clinical response and remission in patients with moderate-to-severe ulcerative colitis. Gastroenterology 2014; 146: 85-95.

17. Sandborn WJ, Feagan BG, Marano C, et al. Subcutaneous golimumab maintains clinical response in patients with moderate-to-severe ulcerative colitis. Gastroenterology 2014; 146: 96-109.

Received: 5.06 .2015

Accepted: 25.06.2015 